\title{
Assessing Effectiveness of a Chiropractic Adjustment for an Individual Patient: A Case Study in Methodology
}

\author{
John Hart, DC, MHSc \\ Greenville, South Carolina USA \\ *Corresponding author: John Hart; jhartdc@yahoo.com \\ Received 03 January 2021; $\quad$ Accepted 19 January 2021; \\ Published 01 February 2021
}

\section{Summary}

Effectiveness of an intervention can be assessed at the level of the individual patient by comparing instances of progress with the intervention versus instances of progress where the intervention was not used. This case report presents a method used for such determination for an individual chiropractic patient.

\section{Keywords: Natural history, heart rate variability, chiropractic spinal adjustment}

\section{Introduction}

The effectiveness of an intervention (treatment) is often determined at the group level involving patients other than the one being seen by the clinician. Another approach is to compare the patient to him or herself. Even though an intervention may show improvement following its delivery, the improvement may occur in other instances in the patient's life without the intervention, as a matter of natural history or self-limited condition. This case study presents a novel method of using the patient's own data as a reference for determining effectiveness of a chiropractic adjustment for that patient.

A core objective in chiropractic care is to improve the health of the nervous system, including the autonomic nervous system, through spinal adjustment ${ }^{[1]}$. Heart rate variability (HRV) is an evidence-based method of assessing autonomic health ${ }^{[2]}$. This is important because healthy autonomic function plays a key role in promoting a long and healthy life ${ }^{[2]}$. Consequently, HRV is an important clinical tool for assessing autonomic progress in the chiropractic patient. Moreover, there is emerging evidence at the group level that chiropractic care is followed by improved HRV ${ }^{[3]}$.

The method used in this study compares HRV in instances of chiropractic spinal adjustment versus instances where no adjustment was given, using the patient as his own reference. In this way, the natural history of HRV change can be the reference for determining efficacy of the intervention. The method may be of interest to other clinicians who would like to assess the effectiveness of their interventions by comparing to the individual patient's natural history.

\section{Case Study}

The author is a chiropractor as well as the patient in this study. The reason for him being the patient in the study is: a) the large amount of HRV data he has accumulated on himself from daily measurements, and b) it seems appropriate for researchers to "testdrive" novel methods on themselves first, before applying it to their patients.

HRV was measured with the App, Heart Rate Variability Logger ${ }^{[4]}$. The App uses an earlobe sensor that sends a Bluetooth signal to the App on a smartphone. The set-up has good agreement with standard ECG technology ${ }^{[5-6]}$. The HRV metric used was the root mean square of successive differences of time between heartbeats (rMSSD). The terms HRV and rMSSD are used interchangeably in this case study. A larger rMSSD number is considered neurologically healthier than a smaller number ${ }^{[2]}$.

HRV was self-measured almost every day beginning 1-120 through $10-15-20$ for a total of 272 measurements. The author received a spinal adjustment on 10-9-20 when the HRV was relatively low, at 26.1 milliseconds (ms). His average HRV in 2020 prior to the adjustment was $36.5 \mathrm{~ms}$. Following the adjustment, his HRV improved (increased) noticeably on six consecutive days. Not content to simply call it good, he wanted to at least compare to other instances of similarly low HRV where no intervention (no spinal adjustment) was given. In this way, a cornerstone of epidemiological investigation can be applied, that of comparison, in this case at the level of the individual patient.

HRV was sorted in Excel (Microsoft Corp) from lowest-tohighest HRV. A millisecond up and down from the aforementioned $26.1 \mathrm{~ms}$ (25.1 ms to $27.1 \mathrm{~ms}$ ) was the inclusion criteria for comparison with the spinal adjustment result. Each instance within this range was identified and considered as a low Baseline reading. The mean of six subsequent and consecutive daily HRV readings is referred to as Subsequent readings (subsequent to a given baseline). The number of Subsequent readings, 6 , was chosen to be consistent with the number after the 10-9-20 spinal adjustment that showed substantial improvement. The Baseline was subtracted from the average of the Subsequent values to arrive at the Difference 
between Baseline and Subsequent, as shown in this example of mock data for one instance of a low Baseline reading:

\begin{tabular}{lll}
\hline Reading type & Date & HRV value (ms) \\
\hline Baseline reading & $1-1-19$ & 25.1 \\
& & \\
Subsequent reading \#1 & $1-2-19:$ & 26.1 \\
Subsequent reading \#2 & $1-3-19$ & 27.1 \\
Subsequent reading \#3 & $1-4-19$ & 28.1 \\
Subsequent reading \#4 & $1-5-19$ & 29.1 \\
Subsequent reading \#5 & $1-6-19$ & 30.1 \\
Subsequent reading \#6 & $1-7-19:$ & 31.1 \\
Mean of subsequent readings: & & 28.6 \\
\hline
\end{tabular}

In this example, the Difference is calculated with, Subsequent (28.6) - Baseline $(25.1)=$ Difference of a 3.5 increase (improvement) in HRV.

\section{Results}

There were seven instances of low baseline HRV (that were between 25.1 and $27.1 \mathrm{~ms}$ ). All instances showed improvement (increases) in Differences, with some showing more improvement than others (Table 1, Figure 1). Average HRV was $26.1 \mathrm{~ms}$ for Baseline, $38.5 \mathrm{~ms}$ for Subsequent, and $12.4 \mathrm{~ms}$ for Difference (Table 1).

Table 1: Summary statistics

\begin{tabular}{llll}
\hline Date & Baseline & Subsequent & Differenc \\
\hline $1 / 6 / 20$ & 26.0 & 30.9 & 4.9 \\
$2 / 17 / 20$ & 26.0 & 40.3 & 14.3 \\
$3 / 11 / 20$ & 26.1 & 29.4 & 3.3 \\
$5 / 18 / 20$ & 26.7 & 42.7 & 16.0 \\
$6 / 9 / 20$ & 25.7 & 47.3 & 21.6 \\
$7 / 20 / 20$ & 26.4 & 29.0 & 2.6 \\
$10 / 9 / 20$ (Adj) & 26.1 & 50.1 & 24.0 \\
Mean & 26.1 & 38.5 & 12.4
\end{tabular}

Baseline is one low HRV reading. Subsequent is the mean of six subsequent readings (that followed the baseline reading). Difference is the difference between baseline and subsequent. "Adj" is spinal adjustment, given on 10-9-20.

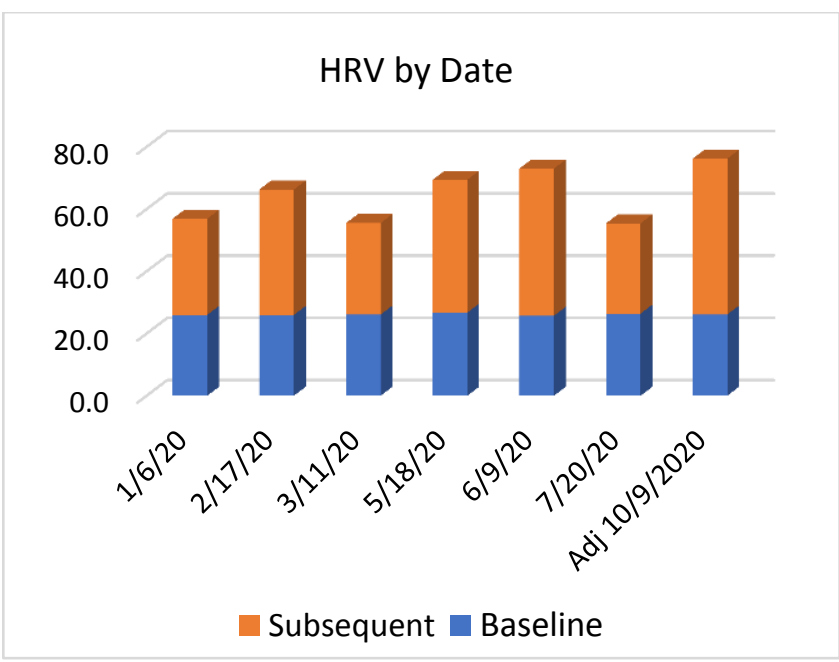

Figure 1: Baseline and Subsequent HRV values (in chronological order)

“Adj” is spinal adjustment. Larger Differences between Baseline and Subsequent readings are shown with taller orange columns (greater improvements).
To better compare differences between the spinal adjustment instance and the six other instances where no-adjustment was received, Differences were sorted from lowest to highest and charted in Figure 2.

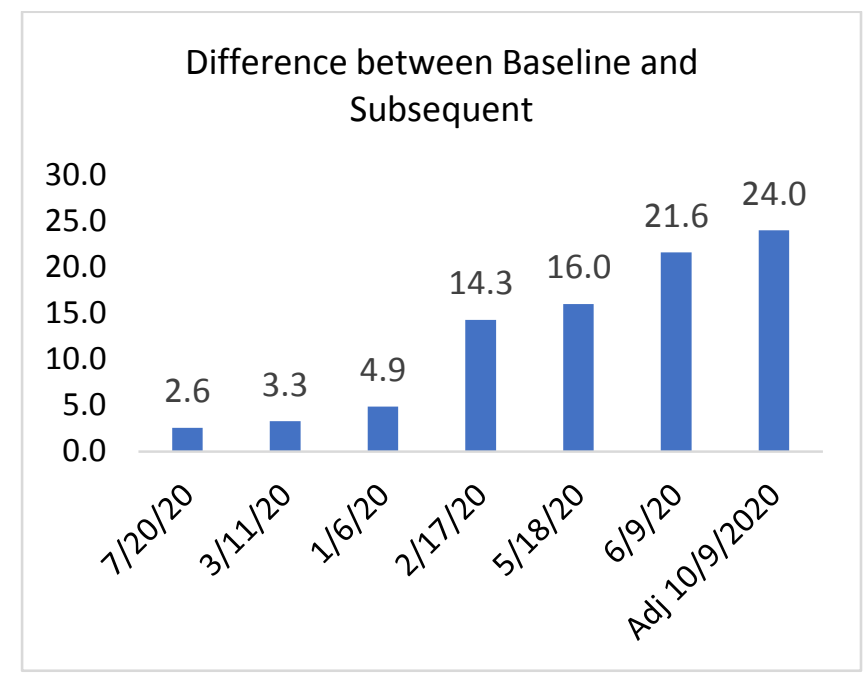

Figure 2: Differences sorted from smallest to largest

Differences between Baseline and Subsequent HRV readings. "Adj" is spinal adjustment.

The Difference for adjustment shows up as the last data point on the far right, with $24.0 \mathrm{~ms}$, indicating that it has the largest (best) Subsequent mean among all seven instances (Figure 2). However, this value, 24.0, was not statistically unusual since it was not an outlier according to the inter-quartile method of moderate outlier detection, where the factor of 1.5 was multiplied by the interquartile range.

\section{Discussion}

Strengths of the study include: a) the amount of data that allows for the comparisons, thanks to daily self-measurements; b) the findings directly apply to the level of the individual patient, using individual data as the reference; and c) the method of comparison may be useful in other health care disciplines to assess their interventions.

A limitation to the study is that few people take daily measurements over long periods of time that are then entered into a spreadsheet. Still, it may be a worthwhile goal for clinicians to teach patients to take self-measurements if the test in question is sufficiently user-friendly, and if there is interest to assess the effectiveness of their interventions. Another limitation is that the best improvement was not an outlier. A stronger case could be made for the spinal adjustment had it been an outlier.

\section{Conclusion}

In this case study, the spinal adjustment on 10-9-20 showed the best improvement in HRV in the Subsequent readings compared to the natural history of HRV change. Further application in other patients willing to collect data on themselves, is a reasonable next step.

\section{List of abbreviations}

HRV: Heart rate variability

rMSSD: Root mean square of successive differences between heartbeats

MS: milliseconds 


\section{Data Availability}

Data is available from the author by request.

\section{Conflicts of Interest}

There are no conflicts of interest

\section{Funding Statement}

No funding was provided for the study.

\section{Authors' contributions}

The author collected and analysed the data, and wrote the manuscript

\section{Bibliographies}

[1] Palmer College of Chiropractic. Philosophy Statement. Retrieved 1-1-19 from: http://www.palmer.edu/aboutus/identity/\#Practice_Paradigm
[2] Usman Z, et al. Relation of high heart rate variability to healthy longevity. The American Journal of Cardiology 2010; 105(8):1181-1185.

[3] Zhang J, Dean D, Nosco D, Strathopulos D, Floros M. Effect of chiropractic care on heart rate variability and pain in a multisite clinical study. Journal of Manipulative and Physiological Therapeutics 2006; 29:267-274.

[4] Altini M. Heart rate variability Logger - app details. December 12, 2013. Accessed 1-1-19 at: https://www.marcoaltini.com/blog/heart-rate-variabilitylogger-app-details

[5] Plews DJ, Scott B, Altini M, Wood M, Kilding AE, Laursen PB. Comparison of heart rate variability recording with smart phone photoplethysmographic Polar H7 chest strap and electrocardiogram methods. International Journal of Sports Physiology and Performance 2017; 12(10)1-17.

[6] Vescio B, Salsone M, Gambardella A, Quattrone A. Comparison between electrocardiographic and earlobe pulse photoplethysmographic detection for evaluating heart rate variability in healthy subjects in short- and long-term recordings. Sensors 2018; 18: 844; doi: 10.3390/s18030844. 